\title{
Context-Aware Process Modelling for Medicinal Product Development
}

\author{
Citation for published version (APA):
}

Ozturk Yurt, Z., Eshuis, R., Wilbik, A., \& Vanderfeesten, I. T. P. (2021). Context-Aware Process Modelling for Medicinal Product Development. In E. Serral, J. Stirna, J. Ralyté, \& J. Grabis (Eds.), The Practice of Enterprise Modeling (PoEM 2021): 14th IFIP WG 8.1 Working Conference, PoEM 2021, Riga, Latvia, November 24-26, 2021, Proceedings (Vol. 432, pp. 168-183). Springer, Cham. Lecture Notes in Business Information Processing Vol. 432 https://doi.org/10.1007/978-3-030-91279-6_12

\section{Document status and date:}

Published: 01/01/2021

DOI:

10.1007/978-3-030-91279-6_12

Document Version:

Publisher's PDF, also known as Version of record

\section{Document license:}

Taverne

\section{Please check the document version of this publication:}

- A submitted manuscript is the version of the article upon submission and before peer-review. There can be important differences between the submitted version and the official published version of record.

People interested in the research are advised to contact the author for the final version of the publication, or visit the DOI to the publisher's website.

- The final author version and the galley proof are versions of the publication after peer review.

- The final published version features the final layout of the paper including the volume, issue and page numbers.

Link to publication

\footnotetext{
General rights rights.

- You may freely distribute the URL identifying the publication in the public portal. please follow below link for the End User Agreement:

www.umlib.nl/taverne-license

Take down policy

If you believe that this document breaches copyright please contact us at:

repository@maastrichtuniversity.nl

providing details and we will investigate your claim.
}

Copyright and moral rights for the publications made accessible in the public portal are retained by the authors and/or other copyright owners and it is a condition of accessing publications that users recognise and abide by the legal requirements associated with these

- Users may download and print one copy of any publication from the public portal for the purpose of private study or research.

- You may not further distribute the material or use it for any profit-making activity or commercial gain

If the publication is distributed under the terms of Article $25 \mathrm{fa}$ of the Dutch Copyright Act, indicated by the "Taverne" license above, 


\title{
Context-Aware Process Modelling for Medicinal Product Development
}

\author{
Zeynep Ozturk Yurt ${ }^{1(\bowtie)}$, Rik Eshuis ${ }^{1}$, Anna Wilbik ${ }^{2}$, \\ and Irene Vanderfeesten ${ }^{1,3}$ \\ 1 Eindhoven University of Technology, Eindhoven, The Netherlands \\ \{z.ozturk.yurt,h.eshuis,i.t.p.vanderfeesten\}@tue.nl \\ 2 Maastricht University, Maastricht, The Netherlands \\ a.wilbik@maastrichtuniversity.nl \\ 3 Open Universiteit, Heerlen, The Netherlands
}

\begin{abstract}
Advanced Therapy Medicinal Products (ATMPs) are highly innovative medicinal products that are based on biomedical technology. ATMP development processes need to comply with complex regulatory frameworks. Currently, biomedical scientists that develop ATMPs manage the regulatory aspects of the ATMP development processes in an ad-hoc fashion, resulting in inefficiencies such as rework, or even withdrawal of ATMPs from the market if the regulatory requirements are not adequately addressed. This paper presents an explorative case study in which we investigate enterprise modelling and context-aware business processes to support ATMP scientists in managing the regulatory aspects of ATMP development processes more efficiently and effectively. The main objective of this case study is to offer regulatory-based guidance to scientists. We use enterprise models (domain, goal and process models) to describe the important concepts and views in ATMP development processes. By introducing context-awareness to the models, we enable regulatory-based guidance that supports ATMP scientists in performing relevant tasks to address the regulatory requirements efficiently and effectively.
\end{abstract}

Keywords: Context-awareness $\cdot$ Enterprise modelling $\cdot$ Business process management $\cdot$ Conceptual modelling

\section{Introduction}

Advanced Therapy Medicinal products (ATMPs) are medicines for human use that are based on innovative biomedical technologies [1]. Being a medicinal product for human use, ATMPs need to comply with complex regulations about safety and efficacy. Therefore, the two most prominent views in ATMP development processes are scientific development and regulatory compliance. Currently, the ATMP scientists manage regulatory aspects of ATMP development processes in an ad-hoc fashion. Yet, ATMP development processes suffer from inefficiencies

(C) IFIP International Federation for Information Processing 2021

Published by Springer Nature Switzerland AG 2021

E. Serral et al. (Eds.): PoEM 2021, LNBIP 432, pp. 168-183, 2021

https://doi.org/10.1007/978-3-030-91279-6_12 
such as reworks and withdrawal of ATMPs from market, due to not being able to adequately demonstrate regulatory compliance [10,18].

The cause of this is the complexity of ATMP regulatory framework and scientists' lack of regulatory knowledge and its impact on the scientific development process. ATMP regulations describe high level goals to be achieved in order to make sure that the ATMP being developed is safe and effective. This is done by, for instance, demonstrating physiological and biochemical properties of the product. Also, ATMP regulations are flexible, depending on development setting different regulatory requirements apply. Here, the development setting covers a set of factors related to the ATMP, defined by the scientist e.g., type of materials used, regulatory classification of materials etc. These two factors make the management of regulatory aspects of ATMP development challenging for scientists. Therefore, there is a need to support ATMP scientists in managing the regulatory aspects more efficiently and effectively.

To enable this support, we first need to investigate the ATMP development setting. Enterprise Modelling is an effective approach to capture, understand and relate the elements of a complex setting [20]. Enterprise modelling can support many purposes, for example, strategy development [15], change management [11] or process improvement [14]. In this case study, we use enterprise modelling as the stepping stone to enable more efficient and effective management of ATMP development processes. Using enterprise models such as: domain, goal and process models, we capture, understand and relate the main elements in ATMP development processes in a structured way. Building upon these models, we focus more on process modelling, in order to investigate ways to provide regulatory-based guidance in the scientific development processes.

Context-aware BPM deals with identifying factors that drive flexibility and variability in business processes [19]. Several authors investigated the notion of context for business processes with an aim to identify factors that affect the design and execution of a business process and make business processes contextaware by integrating these factors and their effect to the process models [21]. In this paper, we use the notion of context-awareness in BPM to guide scientists in working more efficiently and effectively towards regulatory compliance.

This paper presents an explorative case study in which context-aware ATMP development processes are modelled. We use enterprise models to describe scientific and regulatory views in ATMP development. To describe the main concepts and their relations in ATMP development, we use a domain model. We represent the scientific development process in a flexible process model and regulatory requirements in a goal model. By introducing context-awareness, we make the link between different regulatory contexts, regulatory requirements and the scientific development process explicit, and guide scientists in performing relevant tasks to address the regulatory requirements. Thereby, this paper presents an exemplary case study for guiding users in flexible and knowledge-intensive processes towards regulatory compliance.

The object of the case study presented in this paper is the biomaterial development process, which is a part of ATMP development processes, from the Hori- 
zon2020 iPSpine project ${ }^{1}$. In iPSpine, an ATMP for lower back pain is being developed. As a part of this project, we develop a digital platform to enable efficient and effective management of ATMP development processes. Therefore, this case study is driven by the problems in iPSpine.

The remainder of this paper is organized as follows. Section 2 introduces ATMP development processes and the problem addressed in this case study, and presents the objectives of the case study. Section 3 discusses how the objectives of the case study are addressed. Section 4 presents the preliminary evaluation made with iPSpine stakeholders on the usefulness of models and ideas presented in this paper. Section 5 discusses the relevant work on Context-Aware BPM. Lastly, Sect. 6 concludes the paper.

\section{ATMP Development: The Need for Guidance}

In this section, we introduce the ATMP development processes and the problem we address in this case study.

\subsection{ATMP Development Process}

Development of ATMPs involves several stages and the overall aim in these stages is to develop a safe and effective medicinal product. This is accomplished by collaboration of many stakeholders, where scientists and regulatory consultants are the main ones. Figure 1, describes the main phases and stakeholders in ATMP development.

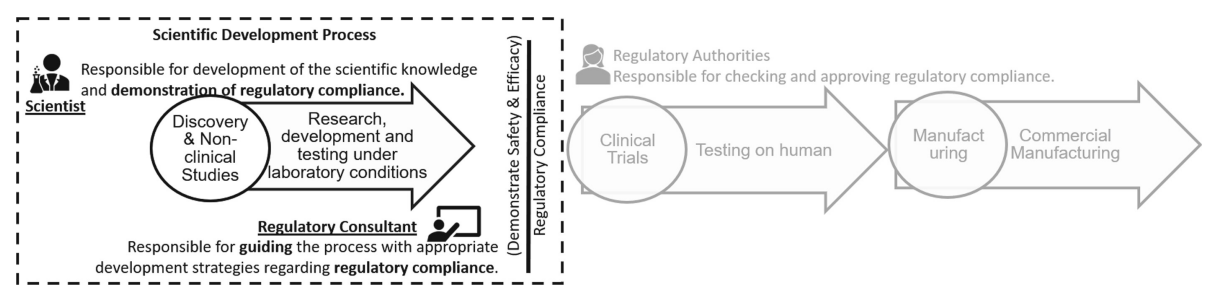

Fig. 1. ATMP development process \& stakeholders (stakeholders and scope of this study in bold)

Research shows that ATMP development processes are associated with many hurdles such as reworks and even withdrawal of the ATMP due to shortcomings in providing adequate evidence for regulatory compliance [10]. This contributes to increased development costs and time-to market. Lack of regulatory knowledge among scientists is an important factor for these hurdles [10]. Being an expert, a scientist requires minimal guidance about the scientific aspects of ATMP development. However, establishing and maintaining the link between the scientific

\footnotetext{
${ }^{1}$ https://ipspine.eu/.
} 
development process and the complex regulatory framework of the ATMP development is challenging for a scientist. In other words, there is a need to bridge the gap between the scientific and regulatory views on ATMP development processes. This requires identification and description of important concepts in an ATMP development setting. To do so, we use the conceptual enterprise models. The following section presents these models.

Note that, for demonstration purposes, we use models from a biomaterial development process, which is a part of the ATMP development studies. The models we use in this paper are simplified for readability and space considerations.

\subsection{Modelling ATMP Development}

Enterprise modelling covers several models $[15,26]$. Depending on the purpose of the enterprise modelling job, the models used and the level of detail included in the models should change [15].

For this case study, the purpose of modelling is to represent and relate the two most prominent views, regulatory and scientific views, of ATMP development processes. The regulatory view covers the reason or motivation behind performing ATMP development processes, i.e. the aim is to develop a safe and effective (in other words, regulatory compliant) product. The scientific view covers the activities to develop the product. Therefore, goal and process models are essential elements for our purpose. To understand and relate different concepts in these different views, a domain model is also essential.

There are other models used in enterprise modelling. For instance, actor/resource models and business rule models [15], organization and network models [26]. However, we haven't used such models since they do not provide considerable information for our modelling purpose. For example, modelling the different actors/resources and their relations do not provide any implications about the scientific and regulatory views in ATMP development, or modelling the business rules, e.g., some scientific procedures that constraint how experiments should be done, is not within the scope of our modelling purpose.

First, we built a domain model with domain experts, to structure the domain knowledge and understand complex concepts and the problems in the domain. Figure 2 shows the domain model we have created for this case study, using UML class diagrams.

ATMP regulations do not induce strict rules on how things should be done throughout the development process. Instead, they involve high-level goals that should be considered in order to demonstrate that the ATMP being developed is safe and effective. Therefore, we represent the regulatory requirements using goal models. Figure 3 shows an excerpt from the goal model of biomaterial development process in GRL notation [23].

Lastly, we model the scientific development process using flexible process models. ATMP development processes are knowledge-intensive processes. Traditional BPM focuses on managing routine and predictable work. Knowledgeintensive processes have different characteristics [9]. Traditional BPM is limited 


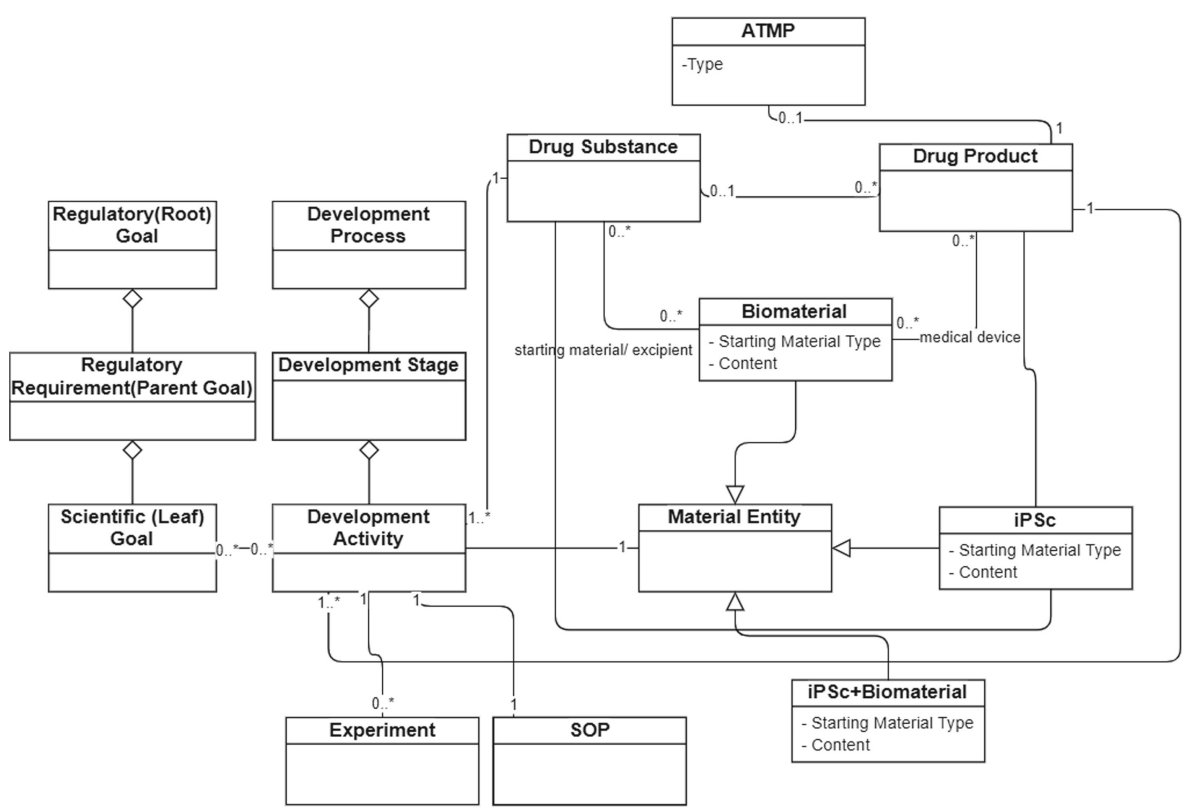

Fig. 2. Domain model of ATMP development

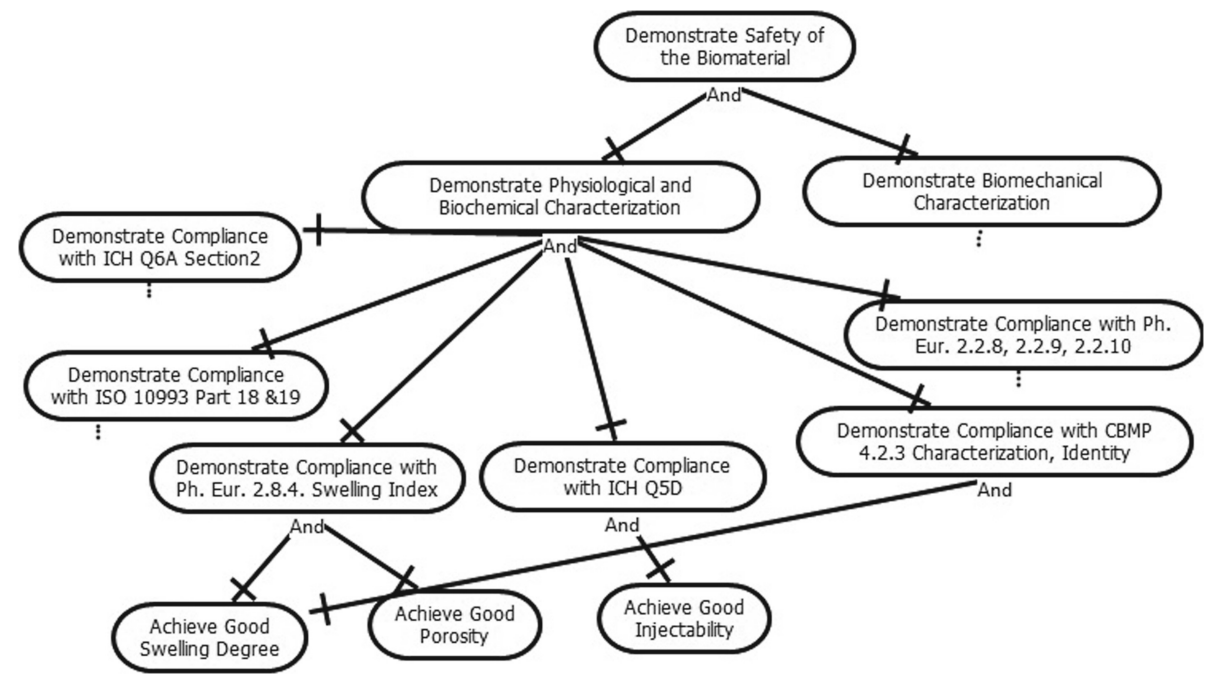

Fig. 3. Goal model of biomaterial development 
when it comes to supporting flexible and unpredictable knowledge-intensive processes [9]. Case Management is an approach that recently emerged to overcome these limitations $[2,22]$. Therefore, we chose to support ATMP development processes with Case Management and, modelled the scientific development process using Case Management Model and Notation (CMMN) [6]. Figure 4 shows an excerpt from the biomaterial development process model.

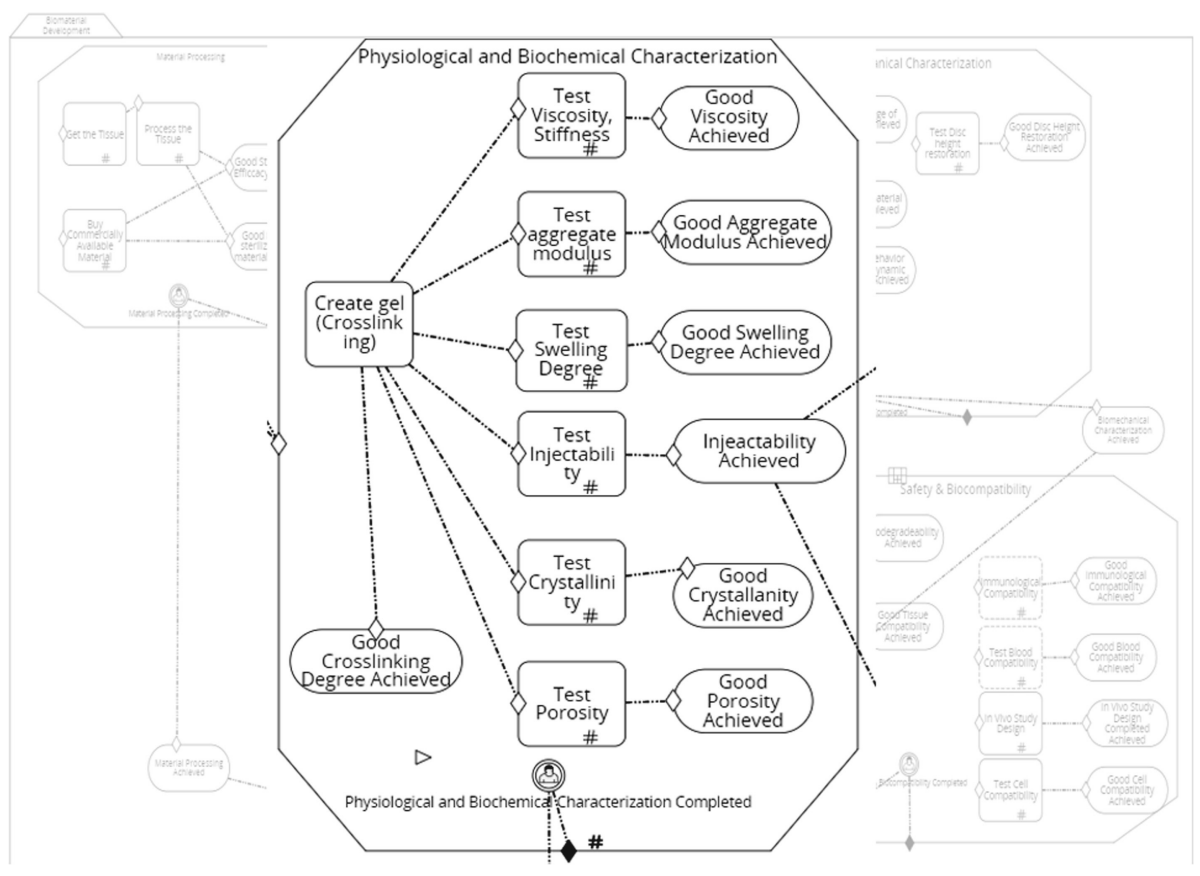

Fig. 4. Process model of biomaterial development

A top-down analysis of regulatory goals results in a goal model where the leaf goals are satisfiable by means of sub-processes or tasks in the process model. This way, we build a link between the regulatory goals and the scientific development process. Each leaf goal in the goal model corresponds to a milestone of a single task or sub-process in the process model. The milestones corresponding to the leaf goals have the same labels as the goals.

\subsection{The Need for Guidance}

Looking at the goal model in Fig. 3, we see that there is a set of sub-goals that are required to achieve Demonstrate Physiological and Biochemical Characterization goal.

Indeed, some factors related to the development process and the ATMP being developed determine which of the sub-goals (regulatory requirements) 


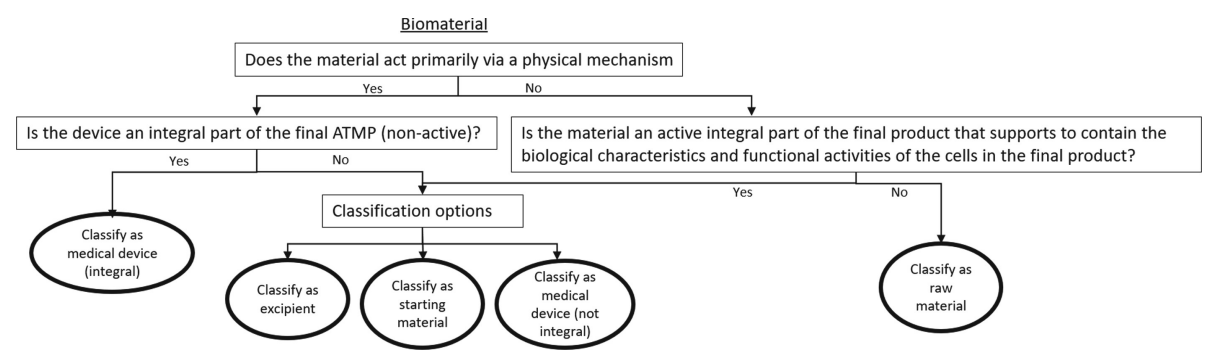

Fig. 5. Decision tree

are required to achieve the Demonstrate Physiological and Biochemical Characterization goal. We refer to a set of such factors as the context of the ATMP development process. For ATMP development, the context is defined by several factors related to the ATMP. For instance, scientist's choice of regulatory classifications for the components of an ATMP or the type of starting material of an ATMP. An example decision tree followed by scientists for regulatory classification decisions is shown in Fig. 5. For different contexts, e.g., different classification options in Fig. 5, different regulatory requirements should be addressed to achieve Demonstrate Physiological and Biochemical Characterization goal. In short, which regulatory requirements are applicable depends on the context.

Correspondingly, since the regulatory goals drive the scientific development process, i.e. the scientist performs experiments to address regulatory requirements, context also affects the scientific development process. The ATMP development process model on Fig. 4 covers all possible tasks a scientist can perform throughout the development process. Yet depending on the context, since context defines which regulatory requirements are applicable, some tasks are required to address the regulatory goals whereas some are not. The scientist can still perform other tasks that are not required to address the regulatory goals of the current context, for instance, out of scientific interest or to explore alternative contexts (See Fig. 5).

The scientist starts the process with an initial assumption on the context. However, the context is subject to changes throughout the development process. For example, different options (e.g., classifying the biomaterial as medical device or excipient) are investigated throughout the development. Depending on the results the scientist obtains throughout the process, she can decide to, for instance, classify the biomaterial as a medical device instead of as an excipient, following the decision tree in Fig. 5. This would change the context, regulatory goals to be addressed and hence the tasks to be executed to address relevant regulatory requirements. To ensure that the scientists performs the relevant tasks that addresses the relevant regulatory requirements, it is important to make explicit on the process model which tasks are required under which conditions (context).

In this case study, we intend to address the need of regulatory guidance in ATMP development processes. As a result of an analysis of literature on manag- 
Table 1. Objectives of the case study

\begin{tabular}{l|l}
\hline Main objective & $\begin{array}{l}\text { Guide the scientists performing the scientific development process } \\
\text { towards relevant regulatory goals }\end{array}$ \\
\hline Sub-objective 1 & Define and represent context for ATMP development processes \\
\hline Sub-objective 2 & Represent the variability of regulatory goals, depending on context \\
\hline Sub-objective 3 & Represent the effect of context on the scientific development process \\
\hline
\end{tabular}

ing ATMP development processes and our interviews with iPSPine stakeholders, we have identified the objectives in Table 1 for our case study.

\section{Solution Design and Development}

The need for regulatory guidance, as discussed in Sect. 2, motivated us to use the notion of context and context-awareness to guide ATMP scientists in working towards regulatory compliance. The following sections present how we address the objectives in Table 1.

\subsection{Contextualizing the Domain Model}

(Sub-objective 1). Every business process has a specific domain. Correspondingly, everything that influences a process is related to this domain [17]. Therefore, what constitutes context for a business process lies in the domain model. This motivates our choice of using domain models as a baseline to define context in ATMP development processes. For ATMP development, the experiments performed, results obtained, properties of the ATMP being developed or decisions taken throughout the process form the context of the development process. For instance, a decision, which is a part of the ATMP development process, about the regulatory classification of components of the ATMP is an important contextual element.

Below is an example (part of the) domain model and context definition. First, we created the domain model with experts. In the domain model, entities and their attributes are marked as contextual, shown in dashed boxes in Fig. 6, if they determine the regulatory goals to be addressed by the development process. For example, the decision about classification of biomaterial shown in Fig. 5, is represented as different roles that a biomaterial entity can take and marked as contextual element (See C1 and C2 in Fig. 6).

Instantiation of each contextual element is a partial context $(\mathrm{C} 5, \mathrm{C} 6, \mathrm{C} 7, \mathrm{C} 1$, $\mathrm{C} 2$ on Fig. 6). Also, combined instantiations of multiple contextual elements with different values is a partial context (C3, C4 on Fig. 6). Contexts which share the same contextual elements but with different values are mutually exclusive (C5, C6, C7 or C1, C2 or C3, C4 on Fig. 6). Contexts which include a combination of multiple contextual elements might imply contexts including less contextual elements (e.g., $\mathrm{C} 4 \rightarrow \mathrm{C} 2, \mathrm{C} 3 \rightarrow \mathrm{C} 2, \mathrm{C} 4 \rightarrow \mathrm{C} 7$ on Fig. 6). So they are not exclusive. 
A set of non-exclusive partial contexts form the overall context in an ATMP development process (See context in Fig. 8).

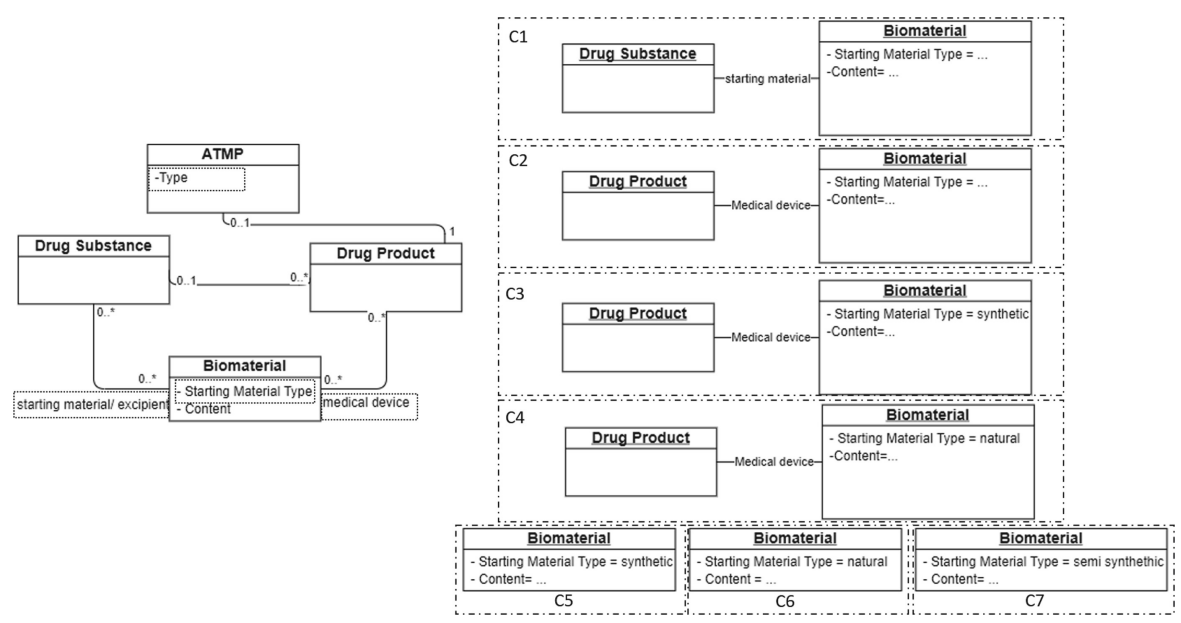

Fig. 6. Domain model (simplified) and example context definitions

\subsection{Contextualizing the Goal Model}

(Sub-objective 2). Having defined contexts, we annotate the root and the parent goals in the goal model with context labels, indicating which goal is adoptable under which conditions. The semantics of context annotations are provided by Ali et al. [3]. If a root goal, $G$, is annotated with a context label $\mathrm{C}_{i}$, that means $G$ is activated iff context $C_{i}$ holds. If there is a goal $G_{i}$, that is decomposed into a sub-goal $\mathrm{G}_{\mathrm{j}}$ with and (or) decomposition links then the link is annotated with a context label. This means, goal $\mathrm{G}_{\mathrm{i}}$, requires (can be achieved) via $\mathrm{G}_{\mathrm{j}}$ iff context $\mathrm{C}_{\mathrm{i}}$ hold.

These annotations enable us to derive the context for leaf goals. Figure 7 shows an example goal model for ATMP development processes where the context for leaf goals are derived using the contexts of goal model variants which includes these leaf goals (See Fig. 8).

The idea of using contextual goal models is inspired from [3]. In [3], authors use contextual goal models to model contextual requirements for an information system. Here, we use contextual goal models as a means to contextualize process models. In the following section, we describe how contextual goal models are used to contextualize ATMP development processes.

\subsection{Contextuzalizing the Process Model}

(Sub-objective 3). Our intention here is to contextualize the process model such that it guides scientists throughout the process execution. This is achieved by 


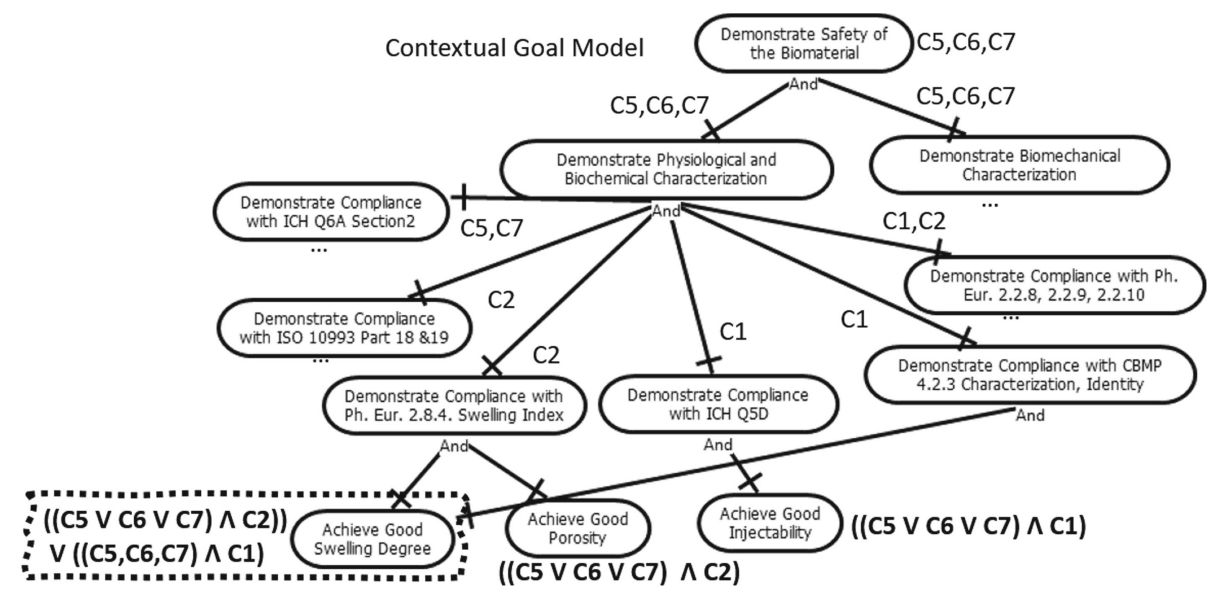

Fig. 7. Contextual goal model

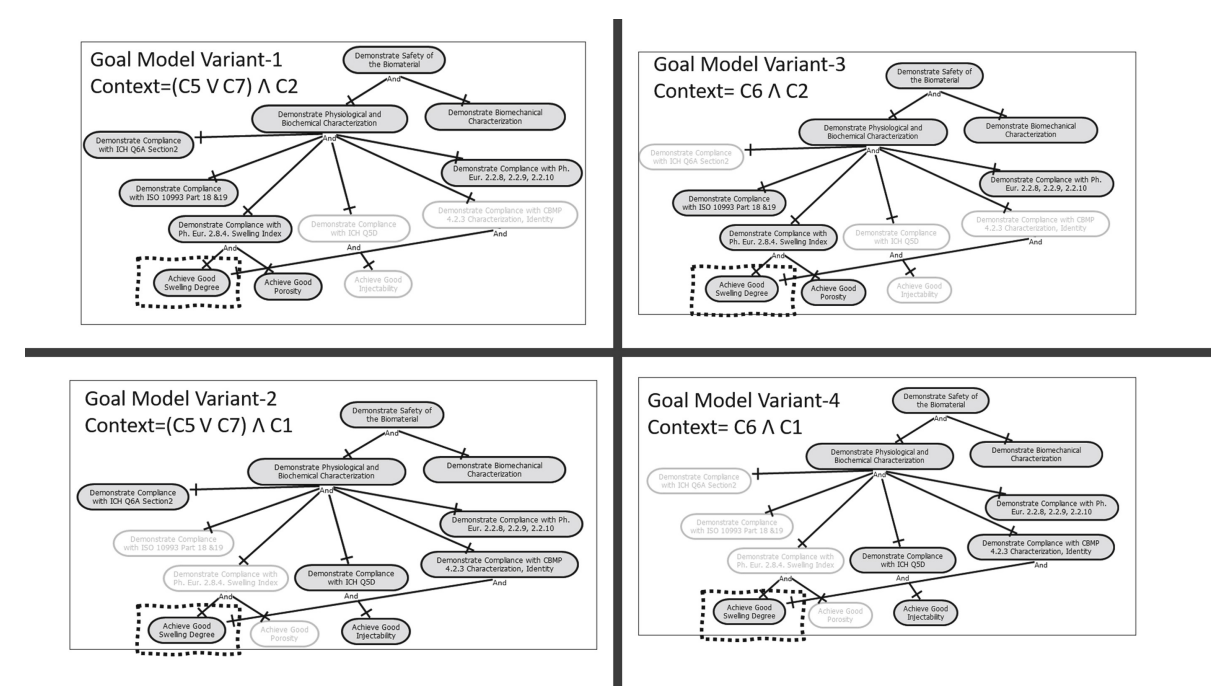

Fig. 8. Goal model variants

deriving the context of the leaf goals in the goal model. A leaf goal corresponds to a milestone of a single task or sub-process in the process model. Accordingly, once we derive the context of leaf goals, the corresponding task/sub-process is also implicitly contextualized. The elements in the domain model used to define the context of its goals are the contextual elements that affect the task or subprocess. The task or sub-process becomes relevant if its context holds. 


\subsection{Guidance Through Context-Aware Process Modelling}

(Main Objective). In this section, using an example, we explain how our models can be used in practice to guide scientists. Context-aware process models support scientists by making explicit which tasks are required to address the regulatory requirements under different conditions(contexts) and what (contextual elements) affects whether a task is required or not. In the following paragraphs, we illustrate how context-aware process models support scientists on an example (Fig. 9).

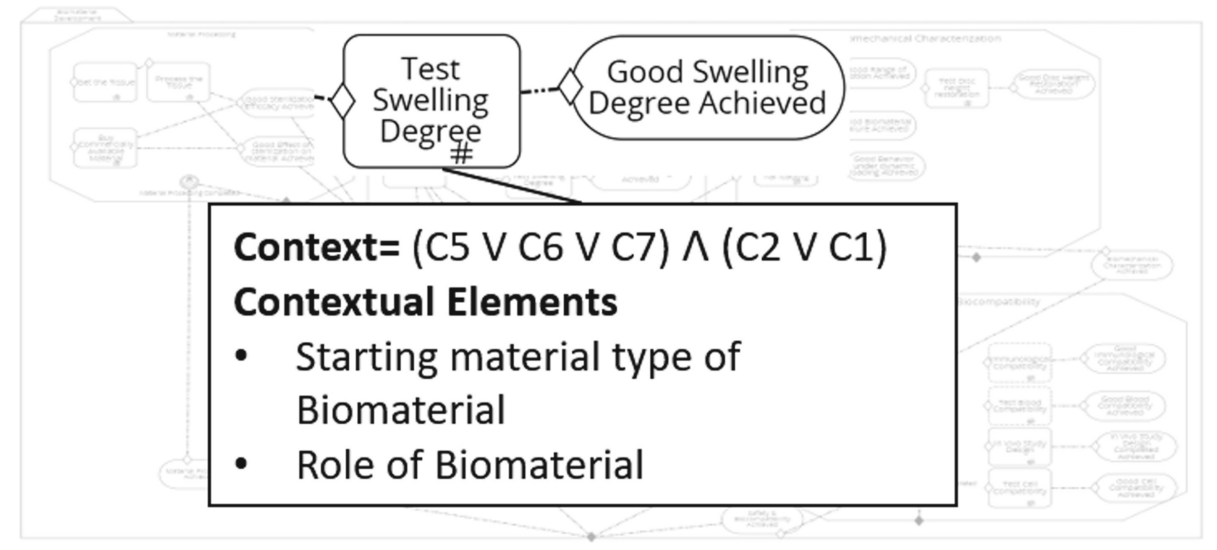

Fig. 9. Contextualized process model

Looking at Fig. 4, we see that once the Create Gel task is completed, a set of tasks are enabled. Being a knowledge-worker, the scientist has the flexibility to choose which tasks to perform and which not. Although this flexibility is an essential part of the process, it is important to support the scientist in making the these choices.

Consider the task Test swelling degree. Without any information on context, the scientist is free to skip this task. However, skipping this task would cause a problem if the biomaterial has a natural starting material and is classified as starting material in the drug substance (context C1 in Fig. 6 holds). Skipping the task will result in not being able to Demonstrate compliance with Ph.Eur. 2.8.4 Swelling Index, (See Fig. 7), and this will result in failing to get the authorizations for clinical trials. With the models in this paper, the scientist can choose a specific context and this way is able to see which goals and tasks are relevant. Thereby, the scientist ensures that the relevant regulatory requirements are addressed.

Additionally, it is important to explicitly show the factors (contextual elements) defining whether a task is relevant for the current context or not. For instance, knowing that the contextual elements related to Test swelling degree task are Starting material type and Role of biomaterial, the scientist sees how Role 
of the biomaterial affects the development process. This helps them define the right context, e.g., choosing the appropriate Role of the biomaterial in Fig. 5, that require fewer tests, which is more time and cost-efficient.

Lastly, since the context can change throughout the development, so do the regulatory goals to be addressed and the set of tasks to be executed to address those regulatory goals. Context-aware process models support scientists by making explicit which tasks are required for which contexts. Thereby, context-aware process models implicitly supports scientists in working more efficiently by helping them in prioritizing the tasks that are relevant for more contexts rather than performing redundant tasks that are only valid for a specific context, which is unlikely to occur.

\section{Preliminary Evaluation}

Initial feedback on the models and ideas presented in this paper has been gathered from senior iPSpine and regulatory experts. The stakeholders indicated that they are positive about the usefulness of models and ideas in practice.

Next, the usefulness of the main-objective of this case study was discussed with three junior scientists who are working on the biomaterial development studies, which is the part of ATMP development processes we focus in this case study. The scientists mentioned that the idea of linking the process model and the goal model is "definitely useful" when the scientific development is at the stage where different regulatory frameworks (different contexts, domain model) are investigated. They mentioned that they can use these models to justify what they have done (process) and identify what they need to do to better comply with the chosen regulatory framework (goals).

Further evaluation will follow when the models are implemented on the process management platform developed within the scope of iPSpine project. This implementation is currently under development. First, we plan to conduct semistructured interviews with junior biomaterial development scientists who are actively using the platform. Then, further interviews will follow with senior scientists and other development cases will be implemented and tested in the platform.

\section{Discussion}

Our focus in this case study is to support scientists in working towards regulatory compliance. We do this by means of context-aware process models. In this regard, the notion of context in context-aware BPM is related to our purpose. Therefore, in this section, we discuss context-aware BPM papers that are highly relevant for our case study.

Context is not a new notion for BPM. Several authors investigated this notion with the aim of making the processes context-aware; responsive to the changes in its environment. Song et al. [21] present a comprehensive survey about various definitions of context in context-aware BPM. The authors conclude that there 
is still a lack of consensus in BPM on how context is defined, represented and integrated to the business processes [21].

A related work on contextualizing business processes is the paper by De La Vara et al. $[7,8]$. They use a context analysis method [3] to contextualize business processes. The context analysis method uses a set of expressions, socalled facts, to check if a particular context holds. A context analysis model defines alternative ways (or alternative combinations of facts) for checking a context, referred to as context variants. In our case, a context can not be verified by facts. Context is defined by different values taken by the contextual elements chosen by the scientist (user).

The importance of goals for investigating and integrating context for business processes is already discovered in context-aware BPM literature $[12,17,19]$. In these papers, goals are used to identify contextual elements, i.e., factors that have an impact on the achievement of business goals, and relate them to the business processes.

Similarly, in this case study, we use goals as a facilitator for identifying and relating context and contextual elements to business processes. However, different from existing approaches $[12,17,19]$, we use contextual goal models for this purpose. In the existing approaches $[17,19]$, analysis of process goals is only limited to identification of top level objectives and discovering factors (contextual elements) that have an effect on the achievement of those goals. Heravizadeh et al. [12], decomposes the process objectives into smaller objectives to discover contextual elements and to link them to the business process. However, in their case, context only affects how or how well the goals are achieved, but the goals are fixed. In our case, different contexts imply different goals.

Another related research area is guidance/recommendations for flexible, knowledge intensive processes. Supporting flexible and knowledge intensive processes is an emerging topic in BPM [9]. Providing guidance and recommendations for those processes has also drawn some attention $[5,13,24,25]$. These approaches provide guidance using historical knowledge about previous cases. ATMP development is a new field with a huge variability between different projects. Also, no historical data from previous projects is available for use. For this reason, existing process guidance approaches are not suitable for our case study. In this regard, this paper presents an exemplary case study for guiding flexible and knowledge-intensive processes where no historical data is available.

Lastly, although business process variability modelling $[4,16]$ is a related research area, it is not the focus of this paper. The main problem we address in this paper was to bridge the gap between the regulatory and scientific views on an ATMP development process. So, our main focus was to identify and integrate the (regulatory related) factors that causes a variability in the scientific development process. Business process variability modelling approaches focus on the next step: deriving different process variants. They do not focus on identification and representation of the factors driving the variability. Also, deriving the process variant for a particular context is not intended in this case study. Alternative contexts are explored throughout an ATMP development study e.g., different 
classification decisions in Fig. 5. So, the process model should cover not only a single process variant of a particular context, but multiple process variants corresponding to alternative contexts that are explored in a single development process.

\section{Conclusion}

In this paper, we have presented a case study on modelling context-aware processes. The immediate contribution of this research are the models presented in Sect. 3 created and implemented for the iPSpine project. These models are used to guide ATMP development scientists work towards regulatory compliance in an efficient and effective manner. Furthermore, this case study presents an exemplary approach for guiding flexible and knowledge-intensive processes through context-awareness.

Contextualization of the existing process model provides a solution for guiding the scientists towards regulatory compliance in this case study. However, ATMP development processes are diverse. The process models we provide in this case study cover only a single ATMP development study in the iPSpine project. As future work, we plan to investigate creating process models, that cover the tasks for a set of contexts explored throughout an ATMP development study, using goal models.

Acknowledgements. The work presented in this paper is part of iPSpine project that has received funding from the European Union's Horizon 2020 research and innovation programme under grant agreement No. 825925 .

\section{References}

1. Advanced Therapy Medicinal Products: Overview. www.ema.europa.eu/en/ human-regulatory/overview/advanced-therapy-medicinal-products-overview. Accessed 05 Oct 2021

2. Aalst, W., Weske, M., Grünbauer, D.: Case handling: a new paradigm for business process support. Data Knowl. Eng. 53, 129-162 (2005)

3. Ali, R., Dalpiaz, F., Giorgini, P.: A goal-based framework for contextual requirements modeling and analysis. Requirements Eng. 15(4), 439-458 (2010)

4. Ayora, C., Torres, V., Weber, B., Reichert, M., Pelechano, V.: VIVACE: a framework for the systematic evaluation of variability support in process-aware information systems. Inf. Softw. Technol. 57(1), 248-276 (2015)

5. Barba, I., Weber, B., Del Valle, C., Jiménez-Ramírez, A.: User recommendations for the optimized execution of business processes. Data Knowl. Eng. 86, 61-84 (2013)

6. BizAgi, et al.: Case management model and notation (CMMN), v1.1, December 2016. OMG Document Number formal/16-12-01, Object Management Group

7. de la Vara, J.L., Ali, R., Dalpiaz, F., Sánchez, J., Giorgini, P.: Business processes contextualisation via context analysis. In: Parsons, J., Saeki, M., Shoval, P., Woo, C., Wand, Y. (eds.) ER 2010. LNCS, vol. 6412, pp. 471-476. Springer, Heidelberg (2010). https://doi.org/10.1007/978-3-642-16373-9_37 